Pacific Journal of Mathematics

ON ORDERS OF TRANSLATIONS AND ENUMERATION

J 


\section{ON ORDERS OF TRANSLATIONS AND ENUMERATIONS}

\section{John Helm, Albert Meyer, and Paul Young}

A basic result of intuitive recursion theory is that a set (of natural numbers) is decidable (recursive) iff it can be effectively enumerated in its natural order (of increasing magnitude). The chief theorems of this paper give simple, but very basic facts relating to enumerations in natural order of magnitude and the extent to which translations must preserve the orders of the sets being enumerated. Under very general conditions for what constitutes a programming system for enumerating the recursively enumerable (r.e.) sets, we prove in Theorem 1 that not every recursive set is "best" enumerated in its natural order, and we later show that under these same general conditions, in every programming system, every recursive set is enumerable in its natural order. We accomplish the latter result by extending Rogers' Isomorphism Theorem to a result which asserts that under these same general conditions, every programming system can be effectively translated into any other in a manner which preserves the order of the sets being enumerated (Theorems 4 and 5). In fact, we show that for every translator, $t$, there is an order preserving pretranslator, $p$, such that $t$ is orderpreserving modulo $p$; i.e., $t \circ p$ is an order preserving translator. In addition, the restriction of the original programming system to the recursive set $\{$ range $p\}$ is a standard programming system on which $t$ is order-preserving. Along the way we establish the existence of sets best enumerated in their natural order and, for every r.e. set, the existence of bad orders for enumerating the set. All proofs are fairly straightforward.

Notation and basic definitions. We let $\lambda x D_{x}$ denote a canonical enumeration of all finite sets (of natural numbers). Given $x$, we can effectively list $D_{x}$ and know when the listing is completed. Except for the indexings $\lambda x W_{x}$ of the r.e. sets which we are about to describe and the notation $W_{x}^{n}$ described later, our notation generally follows [7]. $\langle x, y\rangle$ is (an encoding of) the ordered pair of integers $x$ and $y$.

Definition, ([8]). An 'enumeration technique is a total recursive function $E(x, y)$ such that for every (r.e.) set $W$ there exists an $e$ such that $W=\bigcup_{n} D_{E(e, n)}$. We call $e$ an index of $W$ and denote $\bigcup_{n} D_{E(e, n)}$ by $W_{e}^{E}$, the superscript $E$ being suppressed whenever there is no danger of ambiguity. $D_{E^{\prime}(e, n)}=\bigcup_{m \leqq n} D_{E(e, m)}$.

An enumeration technique is called standard if the $S_{1}^{1}$-theorem is 
satisfied, i.e., if there exists a total recursive function $S$ such that for all $e$ and $x, W_{S(e, x)}=\left\{y \mid\langle y, x\rangle \varepsilon W_{e}\right\}$. We assume without further mention that all enumeration techniques considered in this paper are standard.

The reader should have no trouble convincing himself that all of the usual models for enumerating r.e. sets obviously yield standard enumeration techniques. For example, we might let $D_{E(e, n)}$ denote the set of integers enumerated either in exactly $n$ steps of Turing machine $e$ or in no more than $n$ steps of Turing machine $e$. Clearly, each enumeration technique $E$ partially orders each r.e. set $W_{e}^{E}$ in a natural way which can be extended to a total ordering; that is, we may define $x<_{e}^{E} y$ if $x$ precedes $y$ in the enumeration of $W_{e}^{E}$, and in the case that $x$ and $y$ appear at the same stage of the enumeration we assume that $x$ and $y$ are arbitrarily ordered by some convention uniform in $e$.

Definition. For each enumeration technique $E, x<_{e}^{E} y$ is some fixed r.e. ternary relation of $x, e$, and $y$ such that for each integer $e$,

(i) $<_{e}^{E}$ totally orders $W_{e}$, and

(ii) $\left[p>0\right.$ and $x \in D_{E^{\prime}(e, n)}-D_{E^{\prime}(e, n-1)}$ and $\left.y \in D_{E^{\prime}(e, n+p)}-D_{E(e, n+p-1)}\right] \Longrightarrow$ $\left[x<_{e}^{E} y\right]$.

Clearly, if the binary relations $<_{e}^{E}$ and $<_{e_{0}}^{E_{0}}$ are identical, $W_{e}^{E}=$ $W_{e_{0}}^{E_{0}}$. We shall generally be working with two arbitrary, but fixed enumeration techniques $E$ and $E_{0}$. We abbreviate $W_{e}^{E}$ and $W_{e}^{E_{0}}$ to $W_{e}$ and $W_{e}^{0}$ respectively and similarly $<_{e}^{E}$ and $<_{e}^{E_{0}}$ by $<_{e}$ and $<_{e}$.

DEFINITION. If $E$ and $E_{0}$ are enumeration techniques, a translation from $E$ to $E_{0}$ is a total recursive function $t$ such that $W_{e}=$ $W_{t(e)}^{0}$ for all $e_{0} \quad t$ is order-preserving if $<_{e}=<_{t(e)}^{0}$ for all $e$.

Definition, ([8]). For each enumeration technique $E$, we define $A_{j}(n)=(\mu y)\left[\left|D_{E^{\prime}(i, y)}\right| \geqq n\right]$. If $A_{j}(n)$ exists, $W_{j}^{n}$ is the set consisting of the first $n$ elements of $W_{j}$ in the order $<_{j}$.

This is similar to

Definition, ([1]). For each standard indexing $\lambda i \dot{\phi}_{i}$ of the partial recursive functions, a Blum measure is a r.e. sequence of partial recursive functions $\lambda i \Phi_{i}$ such that for all $i$, domain $\phi_{i}=$ domain $\Phi_{i}$ and the ternary relation $\Phi_{i}(x) \leqq y$ is decidable (under the convention that it is false if $\Phi_{i}(x)$ is undefined).

I. Orders of enumerations. We now prove that any infinite, coinfinite, recursive set has a recursive superset whose natural order is not the best order for enumerating the set. The original proof, [2], was by priority argument, but the proof given here is more in the spirit of [3], and seems to bear evidence for the claim made 
in [3] that "the existence of almost everywhere complex zero-one valued functions is often sufficient to establish the existence of inherently complex computations".

Although the proof we are about to give is largely calculational, the reader will be well-advised to bear in mind that the calculations are merely formal validation of the following intuitive argument: Let $R$ be any infinite and coinfinite recursive set, and let $f$ be a $0-1$ valued function which is much harder to compute than $R$ is to enumerate in increasing order. Let $\bar{r}$ enumerate $\bar{R}$ in increasing order and let $S=R \cup\{\bar{r}(n) \mid f(n)=0\}$. Since $R \subseteq S, S$ can be enumerated almost as easily as $R$, ([10]). But if $S$ can be enumerated easily in natural order, then $S-R$ can also be enumerated easily in natural order. But if this were possible, the following algorithm would give an easy way to compute $f(n)$ : enumerate the first $n+1$ elements of $S-R$; if $\bar{r}(n)$ appears set $f(n)=0$, otherwise $f(n)=1$. We now formalize this in:

THEOREM 1. Let $R$ be an infinite and coinfinite recursive set. Let $\mathscr{F}$ be any effective operator carrying total recursive functions to total recursive functions. Then there exists a recursive set $S$ such that $R \cong S$, and some index $e$ for $S$ such that for any $j$ if $W_{j}=S$ and $<_{j}$ is the increasing order ${ }^{1}$ for $S$, then $\mathscr{F}\left(A_{e}\right)(n)<A_{j}(n)$ for all but finitely many $n$.

Proof. Let $\bar{r}$ be a strictly increasing recursive function with range $(\bar{r})=\bar{R}$. Define $\bar{r}^{-1}$ by $\bar{r}^{-1}(y)=\max \{z \mid \bar{r}(z) \leqq y\}$. (Thus $\bar{r} \bar{r}^{-1}(y) \leqq y$ and $\bar{r}^{-1}(y-1)<y$.) Define a total recursive function $l$ by

$$
W_{l(i)}=R \cup\left\{\bar{r}(n) \mid \dot{\phi}_{i}(n)=0\right\},
$$

and define a very large upper bound, $t$, on the difficulty of enumerating these supersets of $R$ by

$$
t(z)=\max _{i \leqq z}\left\{\mathscr{F}\left(A_{l(i)}\right)(y) \mid \bar{r}^{-1}(y-1)=z\right\} .
$$

Thus for any $i$, for almost all $y$, (a.e., $y$ )

$$
\mathscr{F}\left(A_{l(i)}\right)(y) \leqq t\left(\bar{r}^{-1}(y-1)\right) \text {. }
$$

Define

$$
\dot{\phi}_{s(j)}(y)= \begin{cases}=\text { undefined if } W_{e} \text { has fewer than } \bar{r}(y)+1 \text { elements } \\ =0 & \text { if } \bar{r}(y) \in W_{\jmath}^{\bar{r}(y)+1} \\ =1 & \text { otherwise }\end{cases}
$$

${ }^{1}$ It is well-known for any of the usual enumeration techniques that just a $j$ exists. For an arbitrary enumeration technique, the existence of such a $j$ follows from this fact together with our Theorems 4 and 5 . 
Define

$$
S_{0}(i, n, y) \begin{cases}=\Phi_{s(i)}(n) & \text { if } A_{i}(\bar{r}(n)+1) \leqq y \\ =0 & \text { otherwise }\end{cases}
$$

and

$$
\begin{gathered}
S(n, 0)=S(0, y)=0 \\
S(n, y)=\max _{i \geqq n}\left\{S_{0}(i, n, y), S(n, y-1), S(n-1, y)\right\},
\end{gathered}
$$

so that $S$ is monotone in both variables and enables us to bound the difficulty of computing $\phi_{s(i)}$ in terms of the difficulty of enumerations $W_{i}$ :

$$
\Phi_{s(i)}(n) \leqq S\left(n, A_{i}(\bar{r}(n)+1)\right) \quad \text { a.e., } n \text {. }
$$

We now choose a characteristic function $f$ which is much more difficult to compute than any of the supersets $W_{l(x)}$ of $R$ are to enumerate. Specifically choose a $0-1$ valued total recursive function $f$ such that $\phi_{c}=f$ implies

$$
S(\bar{r}(y)+1, t(y))<\Phi_{c}(y) \quad \text { a.e.; [4], [2] . }
$$

Next note that $\dot{\phi}_{s(j)}$ is so defined that if $j$ enumerates $W_{l(c)}$ in its natural order, then

$$
\dot{\phi}_{s(j)}=f\left(=\phi_{c}\right)
$$

Therefore, by (c),

$$
S(\bar{r}(y)+1, t(y))<\Phi_{s(j)}(y) \quad \text { a.e. }
$$

So

$$
\begin{aligned}
S\left(y, t\left(\bar{r}^{-1}(y-1)\right)\right) & \leqq S\left(\bar{r}\left(\bar{r}^{-1}(y-1)+1\right), t\left(\bar{r}^{-1}(y-1)\right)\right. & & \text { a.e. } \\
& <\Phi_{s(j)}\left(\bar{r}^{-1}(y-1)\right), & & \text { a.e. }
\end{aligned}
$$

By (b),

$$
\begin{array}{rlr}
S\left(y, t\left(\bar{r}^{-1}(y-1)\right)\right) & <S\left(\bar{r}^{-1}(y-1), A_{j}\left(\bar{r}\left(\bar{r}^{-1}(y-1)\right)+1\right)\right) & \text { a.e. } \\
& \leqq S\left(y, A_{j}(y)\right)
\end{array}
$$

Therefore

$$
t\left(\bar{r}^{-1}(y-1)\right)<A_{j}(y) \quad \text { a.e. }
$$

Finally, by (a),

$$
\mathscr{F}\left(A_{l(c)}\right)(y) \leqq t\left(\bar{r}^{-1}(y-1)\right)<A_{j}(y) \quad \text { a.e. }
$$

Although the preceding theorem asserts that in every enumeration technique, not every recursive set is best enumerated in its 
natural order, it is clear that for some models, some recursive sets are best enumerated in their natural order. For example, in Turing machine tape measure, the set $N$ of natural numbers and the set of primes are each best enumerated in natural order of magnitude. In fact, although we omit a proof, for any function $f$, if $f(n)$ is always much bigger than the difficulty of computing $f(0), \cdots f(n-1)$, then the range of $f$ is best enumerated in its natural order.

THEOREM 2. Let $E$ be any enumeration technique and $r$ any total recursive function. There exists a recursive set $R$ with index $e_{0}$ such that $e_{0}$ enumerates $R$ in natural order and for any $j$, and any $n>j$, if $z$ is in $R$ and if $z$ is one of the first $n$ elements of $W_{j}$ (in the order $<_{j}$ ) but is not one of the first $n$ elements of $W_{e_{0}}$, then $r\left(A_{e_{0}}(n), n\right)<$ $A_{j}(n) . \quad$ (Clearly $R$ is best enumerated in its natural order.)

Proof. Define a total recursive function $f$ by defining $W_{f(e)}$ in Stages. At Stage 0 , place 0 into $W_{f(e)}$ and at Stage $n(n \geqq 1)$ do the following: begin computing $A_{e}(n)$; if $A_{e}(n)$ is defined, place into $W_{f(e)}$ the first integer, $y$, such that $y$ is greater than any of the first $n$ elements of $W_{e}$ and for all $j \leqq n$

$$
y \in D_{E^{\prime}(j, z)} \text { implies } z \geqq r\left(A_{e}(n), n\right) .
$$

A quick induction now shows that if we use the recursion theorem to obtain $W_{e_{0}}=W_{f\left(e_{0}\right)}$, then $W_{e_{0}}$ is infinite, and if $y \in W_{e_{0}}$ but $y$ is not one of the first $n$ elements of $W_{e_{0}}$ in the ordering $<_{e_{0}}$ then $y$ is greater than any of the first $n$ elements of $W_{e_{0}}$. Thus $W_{e_{0}}$ is an infinite recursive set and $e_{0}$ must enumerate $W_{e_{0}}$ in increasing order of magnitude.

Furthermore, for any $j$, for any $n \geqq j$, if $y \in W_{e_{0}}$ but $y$ is not one of the first $n$ elements of $W_{e_{0}}$, then we put $y$ into $W_{e_{0}}$ only if $y \in$ $D_{E^{\prime}(j, z)}$ implies $z \geqq r\left(A_{e_{0}}(n), n\right)$. Thus if $y$ is one of the first $n$ elements of $W_{j}$,

$$
\begin{aligned}
A_{j}(n) & \geqq(\mu z)\left[\left|D_{E^{\prime}(j, z)}\right| \geqq n\right] \\
& \geqq(\mu z)\left[y \in D_{E^{\prime}(j, z)}\right] \geqq r\left(A_{e_{0}}(n), n\right) .
\end{aligned}
$$

It is perhaps worth pointing out that Theorem 2 cannot be extended by replacing the arbitrary recursive function $r$ by any very large general recursive operator. To see that this is so, one first defines a total recursive function $\sigma$ such that if $W_{j}$ has at least $2 n$ elements then $W_{\sigma(j)}$ has at least $n$ elements and if $W_{j}$ is infinite $W_{\sigma(j)}=W_{j}$ but $<_{\sigma(j)} \neq<_{j} . \quad\left(W_{\sigma(j)}\right.$ is enumerated by "watching" the elements of $W_{j}$ as they appear and whenever enough have appeared "scrambling" some of them.) Next define a total recursive function 
$h$ by $\left.h^{\prime} j, n, y\right)=A_{o(j)}(n)$ if $y=A_{j}(2 n)$, while $h(j, n, y)=0$ otherwise. Finally define a general recursive operator $\odot$ mapping functions, $t$, to functions by $\mathscr{O}(t)(n)=1+\max _{j \leqq n} h(j, n, t(2 n))$. Now notice that for any infinite r.e. set $W_{i}$ with order $<_{i},<_{i} \neq<_{\sigma(i)}, W_{\sigma(i)}=W_{i}$ and for all $n \geqq i, \mathcal{O}\left(A_{i}\right)(n)>\max _{j \geqq n} h\left(j, n, A_{i}(2 n)\right) \geqq h\left(i, n, A_{i}(2 n)\right)=A_{\sigma(i)}(n)$. Thus the order $<_{i}$ is not " $O$ better" than the order $<_{\sigma(i)}$.

Theorem 2 shows not only that some recursive sets have a best order but also that some orders are (infinitely often) worse than this best order. It is known (although difficult to show) that for some recursively enumerable sets, for every order of enumerating the set there is a much better order, [9]. Whether there are recursive sets with this property appears to be a difficult technical question. On the other hand a simple diagonalization shows that every r.e. set has very bad orders for enumerating the set:

THeorem 3. Given any index e for an infinite r.e. set $S$ and given any total effective operator $\mathscr{F}$, there is an index $j$ for $S$ such that for all $k$, if $W_{k}=S$ and $<_{k}=<_{j}$, then $A_{k}>\mathscr{F}\left(A_{e}\right)$ a.e.

Proof. The index $j$ is determined by the following set of instructions for enumerating a set $B$. (We shall prove that $B=S$.) Let $\lambda i s_{i}$ be some fixed effective enumeration of $S$. To enumerate $B$ at Stage $n(n>0)$ :

1. Find the least $p$ (if any) such that $2 p \leqq n, p$ is not cancelled and $A_{p}(n) \leqq \mathscr{F}\left(A_{e}\right)(n)$. If such a $p$ is found, let $q$ denote the $n$th member of $W_{p}$. Let $r$ be the least number such that $s_{r} \neq q$ and $s_{r}$ is not yet in $B$. Add $s_{r}$ to $B$ and cancel $p$.

2. If no such $p$ is found, add $s_{r}$ to $B$ where $r$ is the least $y$ such that $s_{y}$ has not been added to $B$.

To see that the construction does what is claimed, we note that the intuitive description given above enumerates the set $B$ in a certain order and that we can build a Turing machine which would enumerate $B$ in this very same order. Therefore, by Theorem 4 which follows, there is an index $j$ in the enumeration technique under consideration which enumerates $B$ in the order of the above intuitive enumeration. If $A_{p}(n) \leqq \mathscr{F}\left(A_{e}\right)(n)$ infinitely often, then it is clear that $p$ must be cancelled, and the intuitive description then makes clear that $<_{j} \neq<_{p}$. It now suffices to prove that $B=S$. Clearly $B \subseteq S$, and since Clause 1 can obtain at most $n / 2$ times prior to Stage $n$, Clause 2 must apply infinitely often, forcing $B=S$.

II. Translations. The proof of Theorem 3 used the fact that if a set is enumerable in a given order by Turing machines, it is enumerable in the same order in any enumeration technique. We verify 
this in Theorems 4 and 5 which extend Roger's Isomorphism Theorem to an order isomorphism theorem. The proofs simply extend Rogers' proofs. We first state a well-known (translation) lemma which we then generalize in Theorem 4. We also use a generalization of the recursion theorem.

Lemma 1. (Translation; [6]) Given enumeration techniques $E$ and $E_{0}$, there exists a total recursive function $t$ such that for all $x$, $W_{x}=W_{t(x)}^{0}$.

Proof. $U=\left\{\langle y, x\rangle \mid y \in W_{x}\right\}$ is r.e. so $U=W_{p}^{0}$ for some fixed integer $p$. But $t$ is then just the total recursive function (guaranteed by the $S_{1}^{1}$-theorem for the indexing $\left.\lambda x W_{x}^{0}\right)$ such that $W_{t(x)}^{0}=\{y \mid\langle y, x\rangle \in$ $\left.W_{p}^{\cap}\right\}$.

Lemma 2. (Extended Recursion Theorem) Let $f(x, y)$ be a total recursive function and $E$ and $E_{0}$ arbitrary enumeration techniques. We can effectively find a one-to-one total recursive function $X$ with recursive range such that for all $y,<_{f(X(y), y)}^{0}=<_{X(y)}$.

Proof. For fixed $x$ and $y$, let $i=\langle x, y\rangle, x_{0}, x_{1}, x_{2}, \cdots$ be the members of $W_{x}$ in the order $<_{x}$ and $a_{0}, a_{1}, a_{2}, \cdots$ be the members of $W_{f(x, y)}^{0}$ in the order $<_{f(x, y)}^{0}$. Define the (noncomputable) function $\alpha: N \rightarrow$ $N \cup\{\infty\}$ by $\alpha(i)=\max \left\{z \mid a_{z}\right.$ and $x_{z}$ are both defined and $\left.a_{z}=x_{z}\right\}$, and define the total recursive function $f^{\prime}$ by $W_{f^{\prime}(x, y)}=\left\{a_{0}, a_{1}, \cdots, a_{\alpha(i)}, a_{\alpha(i)+1}\right\}$. That is, $W_{f^{\prime}(x, y)}$ contains the largest initial segment on which $<_{x}$ and $<_{f(x, y)}^{0}$ agree, and in addition $W_{f^{\prime}(x, y)}$ contains the next element, $a_{\alpha(i)+1}$, in the list $<_{f(x, y)}^{0}$ if such an element exists. By the ordinary recursion theorem, there is a one-to-one total recursive function $X$ such that $W_{f^{\prime}(X(y), y)}=W_{X(y)}$ for all $y$.

Clearly if $W_{f^{\prime}(X(y), y)}$ is infinite, we must have $<_{f(X(y), y)}^{0}=<_{X(y)}$. On the other hand, if $W_{f^{\prime}(X(y), y)}$ is finite there are two cases, depending on whether or not $a_{\alpha(i)+1}$ exists. If it does not, then $W_{f^{\prime}(X(y), y)}=$ $\left\{a_{0}, \cdots, a_{\alpha(i)}\right\}$; since $x_{j}=a_{j}$ for all $j \leqq \alpha(i)$, in order to have $W_{f^{\prime}(X(y), y)}=$ $W_{X(y)}$, it is necessary that $W_{X(y)}$ also have only $\alpha(i)$ elements and hence that $<_{X(y)}=<_{f(X(y), y)}^{0}$. If on the other hand, we had $a_{\alpha^{\prime}(i)+1}$ existing, we would similarly have $x_{j}=a_{j}$ for all $j \leqq \alpha(i)$. To have $W_{X(y)}=W_{f^{\prime}(X(y), y)}$ we would also need $x_{\alpha(i)+1}$ to exist. If $x_{\alpha(i)+1} \neq a_{\alpha(i)+1}$ we have the contradiction $x_{\alpha(i)+1} \in W_{X(y)}-W_{f^{\prime}(X(y), y)}$, while if $x_{\alpha(i)+1}=$ $a_{\alpha(i)+1}$ we have, from the difinition of $\alpha(i)$, the contradiction $\alpha(i) \geqq$ $\alpha(i)+1$.

Lemma 2 now enables us to prove a theorem on translations which has interesting interpretations. Suppose we wish to translate from one programming system into another. The most obvious way 
would appear to be to use the programs in the image system to simulate the object programs of which they are the translations. Of course, for a few object programs one may be able to recognize from the program exactly what set the program enumerates and hence translate this program without simulating it, and hence change the orders of enumeration. Similarly if the object program enumerates elements very rapidly, one may be able to have the image program enumerate slowly and "watch" the object program to scramble the order in which the object program enumerates elements. Still, these seem ad hoc methods and one might conjecture that the only general method of translation is simulation. Without saying what it means for one program to simulate another, it does seem clear that if program $p_{0}$ simulates program $p_{1}$ then $p_{0}$ and $p_{1}$ should enumerate the same set in the same order.

Our next theorem guarantees that every translator $t$ is, modulo some order-preserving pretranslator $X$, an order-preserving translator. Alternatively, it guarantees that if we are given enumeration techniques $E$ and $E_{0}$ with a translation $t$ from $E$ to $E_{0}$, then there is a recursive set $\{$ range $X$ \} such that the restriction of the system $E$ to those programs in \{range $X$ \} is a standard enumeration technique $E^{\prime}$, and the fixed translation $t$ translates $E^{\prime}$ to $E$ in an order-preserving way. That is, $t$ is order-preserving modulo some recursive set of programs, $\{\overline{\text { range } X}$, which are not translated by simulation. Finally the theorem guarantees that every two enumerations techniques $E$ and $E_{0}$ can be translated into each other in an order-preserving fashion.

THEOREM 4. (Order-Translation). Given enumeration techniques $E$ and $E_{0}$, and a translation $t$ from $E$ to $E_{0}$, we can effectively find $a$ one-to-one total recursive function $X$ with recursive range such that if we define $G(y)$ to be $t(X(y))$, then $G$ is an order translation: for all $y,<_{y}=<_{G(y)}^{0}$ (and so $W_{y}=W_{G(y)}^{0}$ ). Furthermore $X$ can be so chosen that $G(y)$ always enumerates the elements of $W_{G(y)}^{0}$ one element at $a$ time: i.e., for all $n, D_{E^{\prime}(G(y), n+1)}-D_{E^{\prime}(G(y), n)}$ has at most one element. Finally, $X$ can be so chosen that $<_{y}=<_{X(y)}$ for all $y$.

Proof. Let $y_{0}, y_{1}, y_{2}, \cdots$ the members of $W_{y}$ in the order $<_{y}$ and let $X_{0}, X_{1}, X_{2}, \cdots$ be obtained from the sequence $\lambda n\left[D_{E^{\prime}(t(x), n+1)}-\right.$ $\left.D_{E^{\prime}(t(x), n)}\right]$ by deleting all empty sets. (So $X_{0}, X_{1}, X_{2}, \cdots$ is the natural partial ordering of $W_{x}$ by $t(x)$; so it induces a partial suborder of $<_{t(x)}^{0}$.) We now define a partial recursive function $f_{0}(x, y, n)$ as follows:

$$
f_{0}(x, y, 0)=y_{0}
$$

$f_{0}(x, y, n+1)$ is undefined unless $f_{0}(x, y, n), y_{n}, y_{n+1}$, and $X_{n}$ are all 
defined and $\left\{y_{n}\right\}=X_{n} . f_{0}(x, y, n+1)=y_{n+1}$ if $f_{0}(x, y, n), y_{n}, y_{n+1}$, and $X_{n}$ are all defined and $X_{n}=\left\{y_{n}\right\}$. For each $x$ and $y, \lambda n f_{0}(x, y, n)$ is a computable partial function and by elementary construction there exists a total recursive function $f$ such that for each $x$ and $y, f(x, y)$ is (the Gödel number of) a Turing machine which enumerates the range of $\lambda n f_{0}(x, y, n)$ in the order $<_{s(x, y)}^{T}\left(=f_{0}(x, y, 0), f_{0}(x, y, 1), f_{0}(x, y, 2), \cdots\right)$. By our extended Recursion Theorem, there exists a one-to-one total recursive function $X$ such that $<_{f(X(y), y)}^{T}=<_{X(y)}$ for all $y$.

It is clear from the construction that if range $\lambda n f_{0}(X(y), y, n)$ is infinite, then we must have both $<_{y}=<_{t(X(y))}^{0}$ and $<_{y}=<_{f(X(y), y)}^{T}$ $\left(=<_{X(y)}\right)$, completing the proof. On the other hand if $y_{n}$ is the last element placed into range $\lambda n f_{0}(X(y), y, n)$, there are three possibilities to consider. If $y_{n+1}$ does not exist, then $W_{X(y)}=W_{f(X(y), y)}^{T}=\left\{y_{0}, y_{2}, \cdots, y_{n}\right\}=$ $W_{y}$; since for $m<n$, we must have had $X_{m}=\left\{y_{m}\right\}$, the only way to have $W_{t(X(y))}^{0}=\left\{y_{0}, y_{1}, \cdots, y_{n-1}, y_{n}\right\}$ is to also have $X_{n}=\left\{y_{n}\right\}$, establishing $<_{t(X \mid y)\rangle}^{0}=<_{y}$; since it is clear from the construction that if $y_{n+1}$ does not exist, we must have $<_{f(X(y), y)}^{T}=<_{y}$ and since $<_{f(X(y), y)}^{T}=<_{X(y)}$, this again completes the proof. If $y_{n+1}$ exists but $X_{n}$ is not defined, since $X_{m}=\left\{y_{m}\right\}$ for all $m<n$, we would have that $W_{t(X(y))}^{0}=W_{X(y)}$ would have only $n$ elements while $W_{f(X(y), y)}^{T}$ has $n+1$ elements, contradicting $W_{f(X(y), y)}^{T}=W_{X(y)}$. Finally if $y_{n+1}$ and $X_{n}$ both exist but $X_{n} \neq\left\{y_{n}\right\}$, then $X_{n}$ contains some elements other than $y_{n}$, and since for $m<n, X_{m}=\left\{y_{m}\right\}, X_{n}$ contains some element not in $\left\{y_{0}, \cdots, y_{n}\right\}=$ $W_{f(X(y), y)}^{T}$; this again contradicts $W_{f(X(y), y)}^{T}=W_{X(y)}$.

In closing, we remark that the above proof is easily modified to avoid an appeal to Turing machines and to the recursion theorem, provided we do not require $<_{X(y)}=<_{y}$.

COROLlaRY 1. In every enumeration technique $E$, a set is recursive iff it can be enumerated in natural order of magnitude.

Proof. In the system $E_{0}$ obtained by enumerating sets as ranges of total recursive functions, the result is trivial. It thus follows from Theorem 4.

To give our extension of Rogers' theorem, we first extend his padding lemma:

Lemma 3. (Order Padding) Let $E$ be any enumeration technique and let $e(x)$ be the least member of $D_{x} . \quad\left(e(x)=0\right.$ if $\left.D_{x}=\phi.\right)$ There exists a total recursive function $p$ such that if $D_{x} \neq \phi$ and if $y \in D_{x}$ implies $W_{y}=W_{e(x)}$, then

$$
p(x) \notin D_{x} \quad \text { and } \quad<_{p(x)}=<_{e(x)} \quad\left(\text { and } \quad W_{p(x)}=W_{e(x)}\right) .
$$


Proof. A direct proof which simply extends the ordinary proof of padding implicit in [6] is a straightforward but slightly tedious exercise. However Lemma 3 may also be proven by observing that it is well-known that there are one-to-one translations between any two standard indexings. Theorem 4 now guarantees that the one-toone translations can be made into one-to-one order-preserving translations. Since Lemma 3 is clearly true for any of the well-known enumeration techniques such as Turing machines, it follows directly by translating the problem from $E$ to Turing machines, obtaining the new index for Turing machines, and then translating back to $E$.

Having the Translation Theorem (Theorem 4) and the Padding Lemma (Lemma 3) we can now extend Rogers' Isomorphism Theorem via the usual proof, which we merely indicate.

THEOREM 5. For any two enumeration techniques $E$ and $E_{0}$, there is a recursive permutation $t$ such that $<_{e}=<_{t(e)}^{0}$.

Proof. The function $t$ is constructed in Stages. Stage $2 n$ is used to guarantee that the index $n$ appears in the domain of $t$ : The Translation Theorem (from $E$ to $E^{\prime}$ ) guarantees that we can find an index $n^{\prime}$ such that $<_{n}=<_{n}^{\prime}$, while the Padding Lemma guarantees that we can find an $n^{\prime \prime}$ not yet in the range of $t$ such that $<_{n^{\prime}}^{0}=$ $<_{n^{\prime \prime}}^{0}$, so we define $t(n)=n^{\prime \prime}$. Stage $2 n+1$ is used to guarantee that $t$ is onto by placing $n$ in the range of $t$ : first the Translation Theorem (from $E_{0}$ to $E$ ) is used to find an index $i$ such that $<_{i}=<_{n}^{0}$ while the Padding Lemma (for $E$ ) is then used to find an index $i^{\prime}$ such that $<_{i}=<_{i}$, while $i^{\prime}$ is not yet in the domain of $t$ so that we may set $t\left(i^{\prime}\right)=n$.

Given two enumeration techniques $E$ and $E_{0}$, it is easy to construct translations $t$ from $E$ to $E_{0}$ such that for infinitely many distinct r.e. sets $W$ there are indices $i$ of $W$ such that $<_{i} \neq<_{t(i)}^{0}$. Nevertheless, we conjecture that every translation must preserve "many" orders. For example, we conjecture that for every translation $t$ there are infinitely many r.e. sets $W$ (of cardinality greater than 1 ) such that $W_{i}=W$ implies $<_{i}=<_{t(i)}^{0}$.

As an immediate consequence of Theorem 4, we know that every translation preserves every order infinitely often:

CoROLLARY 1. Let $E$ and $E_{0}$ be enumeration techniques and $t a$ translation from $E$ to $E_{0}$. Then the set $S_{j}$ defined by $S_{j}=\left\{i \mid<_{i}=\right.$ $<_{j}$ and $\left.<_{i}=<_{t(i)}^{0}\right\}$ is not recursive. (It is clearly co-r.e.)

Proof. If $W_{j}$ has at most one element, the corollary reduces to 
the well-known fact that $\left\{i \mid W_{i}=W_{j}\right\}$ is not recursive. Otherwise Theorem 4 guarantees the existence of an $e_{0}$ such that $W_{e_{0}}^{0}=W_{j}$ but $<_{j} \neq<_{e_{0}}^{0}$. Hence if $S_{j}$ were recursive we could obtain a new translation $t^{\prime}$ for which $\left\{i \mid<_{i}=<_{j}\right.$ and $\left.<_{i}=<_{t^{\prime}(i)}^{0}\right\}$ would be empty. It thus suffices to prove that $S_{j}$ is nonempty. But this also follows immediately from Theorem 4.

In a forthcoming paper, "Notes on difficulties of enumerations", we will present some results relating difficulty of enumerations, difficulty of computations, difficulty of decision problems, one-one and many-one reducibilities, and classifications of the r.e. sets.

\section{REFERENCES}

1. M. Blum, A machine-independent theory of the complexity of recursive functions, J. Assoc. Comp. Mach., 14 (1967), 332-336.

2. J. Helm, Two Topics in Recursion Theory, Purdue Univ. Dissertation, 1970.

3. A. Meyer and E. McCreight, Computationally complex and pseudorandom zero-one valued functions, in Theory of Machines and Computations, Academic Press, New York, (1971), 19-42.

4. M. O. Rabin, Degree of difficulty of computing a function, Hebrew Univ. Tech. Report, 2 (1960).

5. W. Ritter, Notation systems and an effective fixed point property, Proc. Amer. Math. Soc., 17 (1966), 390-395.

6. H. Rogers, Gödel numberings of partial recursive functions, J. Symbolic Logic, 23 (1958), 331-341.

7. - Theory of Recursive Functions and Effective Computability, McGraw-Hill, New York, 1967.

8. P. Young, Toward a theory of enumerations, J. Assoc. Comp. Math., 16 (1969), 328-348.

9. - Speed-ups by changing the order in which sets are enumerated, Math. Systems Theory, 5 (1971), 148-156.

10. - Speed-ups by changing the order in which sets are enumerated, preliminary abstract, Proc. 1st Annual ACM Symposium on Theory of Computing, ACM, New York (1969), 89-92.

Received February 23, 1972 and in revised form October 9, 1972. The first author was partially supported by NSF Research Grant GP-6120, Purdue University. The second author was partially supported by Project MAC, an MIT Research Program sponsored by the Advanced Research Projects Agency, Dept. of Defense, Office of Naval Research Contract Number NONR $4102(01)$. The third author was partially supported by NSF Research Grant GJ-27127, Purdue University and GJ-596, University of Hawaii. Reproduction in whole or in part is permitted for any purpose of the United States Government.

\section{Massachusetts Institute of Technology}

Present address for Paul Young: Visiting Professor, Electrical Engineering and Computer Sciences Department, University of California, Berkeley, California, 94720. 



\section{PACIFIC JOURNAL OF MATHEMATICS}

\section{EDITORS}

D. Gilbarg and J. Milgram Stanford University

Stanford, California 94305

R. A. Beaumont

University of Washington

Seattle, Washington 98105
J. DugundJI

Department of Mathematics

University of Southern California

Los Angeles, California 90007

RICHARD ARENS

University of California

Los Angeles, California 90024

ASSOCIATE EDITORS
E. F. BECKENBACH
B. H. NeumanN
F. WOLF
K. YoSHIDA

\section{SUPPORTING INSTITUTIONS}

UNIVERSITY OF BRITISH COLUMBIA

CALIFORNIA INSTITUTE OF TECHNOLOGY

UNIVERSITY OF CALIFORNIA

MONTANA STATE UNIVERSITY

UNIVERSITY OF NEVADA

NEW MEXICO STATE UNIVERSITY

OREGON STATE UNIVERSITY

UNIVERSITY OF OREGON

OSAKA UNIVERSITY
UNIVERSITY OF SOUTHERN CALIFORNIA

STANFORD UNIVERSITY

UNIVERSITY OF TOKYO

UNIVERSITY OF UTAH

WASHINGTON STATE UNIVERSITY

UNIVERSITY OF WASHINGTON

$\stackrel{*}{*} \stackrel{*}{*} \stackrel{*}{*}$ AMERICAN MATHEMATICAL SOCIETY

NAVAL WEAPONS CENTER 


\section{Pacific Journal of Mathematics}

\section{Vol. 46, No. $1 \quad$ November, 1973}

Allan Francis Abrahamse, Uniform integrability of derivatives on $\sigma$-lattices .......................................... 1

Ronald Alter and K. K. Kubota, The diophantine equation $x^{2}+D=p^{n} \ldots \ldots \quad 11$

Grahame Bennett, Some inclusion theorems for sequence spaces .......... 17

William Cutler, On extending isotopies ........................ 31

Robert Jay Daverman, Factored codimension one cells in Euclidean

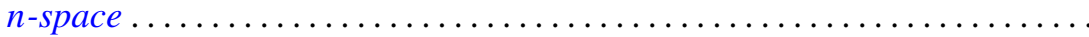

Patrick Barry Eberlein and Barrett O’Neill, Visibility manifolds ............ 45

M. Edelstein, Concerning dentability .......................... 111

Edward Graham Evans, Jr., Krull-Schmidt and cancellation over local

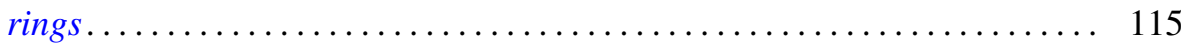

C. D. Feustel, A generalization of Kneser's conjecture ................ 123

Avner Friedman, Uniqueness for the Cauchy problem for degenerate parabolic equations .......................................... 131

David Golber, The cohomological description of a torus action ............ 149

Alain Goullet de Rugy, Un théorème du genre "Andô-Edwards" pour les

Fréchet ordonnés normaux..............................

Louise Hay, The class of recursively enumerable subsets of a recursively enumerable set ........................................

John Paul Helm, Albert Ronald da Silva Meyer and Paul Ruel Young, On orders of translations and enumerations...

Julien O. Hennefeld, A decomposition for $B(X)^{*}$ and unique Hahn-Banach

extensions

Gordon G. Johnson, Moment sequences in Hilbert space .

Thomas Rollin Kramer, A note on countably subparacompact spaces ...

Yves A. Lequain, Differential simplicity and extensions of a derivation ....

Peter Lorimer, A property of the groups Aut $\mathrm{PU}\left(3, q^{2}\right) \ldots$

225

Yasou Matsugu, The Levi problem for a product manifold.

231

John M.F. O'Connell, Real parts of uniform algebras ......

William Lindall Paschke, A factorable Banach algebra without bounded

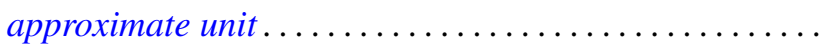

Ronald Joel Rudman, On the fundamental unit of a purely cubic field ....

Tsuan Wu Ting, Torsional rigidities in the elastic-plastic torsion of simply connected cylindrical bars .........................

Philip C. Tonne, Matrix representations for linear transformations on analytic sequences...................................

Jung-Hsien Tsai, On E-compact spaces and generalizations of perfect

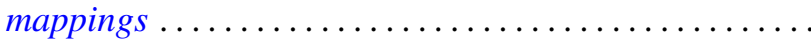

Alfons Van Daele, The upper envelope of invariant functionals majorized by an invariant weight. .. 